\title{
Stochastic Learning Solution for Constrained Nash Equilibrium Throughput in Non Saturated Wireless Collision Channels
}

\author{
Essaid Sabiri, 1 \\ ${ }^{1}$ LIA/CERI, University of \\ Avignon, Agroparc BP 1228 \\ Cedex 9, Avignon, France \\ essaid.sabir@univ- \\ avignon.fr
}

\author{
Rachid El-Azouzi \\ ${ }^{1}$ LIA/CERI, University of \\ Avignon, Agroparc BP 1228 \\ Cedex 9, Avignon, France \\ rachid.elazouzi@univ- \\ avignon.fr
}

\author{
Veeraruna Kavitha ${ }^{1,3}$ \\ ${ }^{3}$ MAESTRO, INRIA \\ Sophia Antipolis, France \\ Kavitha.Voleti Veeraruna \\ @sophia.inria.fr
}

\author{
Yezekael Hayel \\ ${ }^{1}$ LIA/CERI, University of \\ Avignon, Agroparc BP 1228 \\ Cedex 9, Avignon, France \\ yezekael.hayel@univ- \\ avignon.fr
}

\author{
El-Houssine Bouyakhf \\ ${ }^{2}$ LIMIARF, University of \\ Mohammed V-Agdal \\ B.P. 1014 RP, Rabat, Morocco \\ bouyakhf@mtds.com
}

\begin{abstract}
We consider finite number of users, with infinite buffer storage, sharing a single channel using the aloha medium access protocol. This is an interesting example of a non saturated collision channel. We investigate the uplink case of a cellular system where each user will select a desired throughput. The users then participate in a non cooperative game wherein they adjust their transmit rate to attain their desired throughput. We show that this game, in contrast to the saturated case, either has no Nash Equilibrium or has infinitely many Nash Equilibria. Further, we show that the region of NE coincides with an appropriate 'stability region'. We also discuss the efficiency of the equilibria in term of energy consumption and congestion rate. Next, we propose two learning algorithms using a stochastic iterative procedure that converges to the best Nash equilibrium. For instance, the first one needs partial information (transmit rates of other users during the last slot) whereas the second is an information less and fully distributed scheme. We approximate the control iterations by an equivalent ordinary differential equation in order to prove that the proposed stochastic learning algorithm converges to a Nash equilibrium even in the absence of any coordination or extra information. Extensive numerical examples and simulations are provided to validate our results.
\end{abstract}

\section{Keywords}

QoS, Collision channel, Constrained Nash equilibrium, Stability, Throughput.

\section{INTRODUCTION}

Aloha [4] and slotted Aloha [5] have long been used as random distributed medium access protocols for radio channels. They are used in satellite networks and cellular telephone systems for the sporadic transfer of data packets. Modern wireless networks protocols are often based on Aloha-related concept. For our analysis, we use the standard slotted Aloha model. Slotted Aloha and its unslotted version (pure Aloha) has been central to the understanding of random access networks. These two protocols have over the years evolved into a rich family of medium access control schemes, most notably CSMA/CD, the Ethernet standard, and CSMA/CA which is the basis of the IEEE 802.11 protocol. All results presented in this paper are easily extended to CSMA and CSMA/CD.

A major challenge in designing such protocol is how to provide quality of service (QoS) guarantees to various multimedia applications. Quality of Service (QoS) is defined as the ability to provide a level of assurance for data delivery over the network. Hence the required throughput of a node may be dictated by its application (such as video or voice).

In this paper we reconsider the model suggested in [9]. We consider a shared uplink in the form of a collision channel, where a users's transmission can be successful only if no other user attempts transmission simultaneously. Packets those are involved in a collision are backlogged and are scheduled for retransmission after a random time. The determination of the above random time can be considered as a stochastic control problem. We study this control problem in a noncooperative framework: each user has a fixed throughput demand and it dynamically adapts its transmission probability in order to obtain its required demand. For such game, we need to use an important concept, namely, a Nash equilibrium ${ }^{1}$. The paper [9] studied the propriety

\footnotetext{
${ }^{1} \mathrm{~A}$ Nash equilibrium is a strategies profile where no player can improve its reward by deviating unilaterally from its
} 
of Nash equilibrium (NE) under saturated case, i.e., each node has always packets ready to send. Unfortunately, the saturation assumption is unlikely to be valid in most real networks. Data traffic such as web browsing and email are typically bursty in nature while streaming traffic such as voice operates at relatively low rates and often in an on-off manner. Hence, for most real traffic the demanded transmission rate is variable with significant idle periods. In [22], authors assumed that users are independently active with some given probability. Our first main result is then to show the inaccuracy of the independence assumption used those latter works. Our second aim is to derive a mathematical model that allows us to study an important example of non saturated case, the slotted aloha system with infinite buffer queues. More precisely, a user has in general a limited information to transmit, hence it will stop using network when it succeeds all its transmissions. In this paper, we analyze the system equilibria without this assumption. We consider a more realistic model in which users transmit only during their activity period. Yet the activity duration depends on the volume of information the user needs to send and the required transmit rate. We show that this new approach enlarges the existence condition of the Nash equilibrium. In contrast to [9] for this non saturated case we establish the possible existence of infinitely many NE.

In addition to discussing some properties of the Nash equilibrium, we also propose two discrete stochastic learning transmission control algorithms which converge to a Nash equilibrium. The first one is based on the Best Response algorithm in which all users iteratively updates their transmission probability through a given rule. But in this algorithm, all users should be able to obtain good estimates of the idle probability . We finally propose another learning algorithm using a stochastic iterative procedure. We approximate the control iterations by an equivalent ordinary differential equation (ODE) to prove that the proposed stochastic learning algorithm converges to a Nash equilibrium even in the absence of any extra information.

Related works-Interest has been growing in recent years in studying competition of networking in general, access to a common medium in particular, within the frame of noncooperative game theory, see e.g. the survey paper [8]. Various game formulations of the standard Slotted Aloha (with a single power) have been derived and studied in $[7,6,12$, $13,17]$ for the non-cooperative choice of transmission probabilities. In $[7,6,2]$ consider slotted Aloha system as both cooperative (where a common objective is jointly optimized) and non cooperative game, where each users fine-tunes its transmit probability to maximize its payoff, with partial information and power diversity. In [17], the authors discuss the equilibrium of a non-cooperative game for Aloha protocols. In their game formulation, Users are heterogeneous and each one fine-tunes its transmit probability in order to guarantee its demand. The work in [13] discusses the stability of slotted Aloha with selfish users behavior and perfect information. Authors showed the existence of an equilibrium and characterized it.

A stochastic learning technique has been successfully used in wireless network $[19,14,3]$. In [14], the authors propose a

current strategy. stochastic learning algorithms for distributed discrete power control game in wireless network. At each iteration, the only information needed to update the power strategies for individual terminal users is the feedback (payoff) from the base station. The convergence and stability of the learning algorithm are theoretically studied in detail for a two-user two-power-level case.

Paper organization- We start by formulating the problem in Section 2. We analyze the stability region and throughput equilibrium in Sections 3 and 4 . Then, we propose some mechanisms to converge to the best NE in Section 5. Next, we provide extensive numerical illustrations and simulation examples to valid our analytical results. And finally finish our paper by some concluding remarks. More details and some proofs are omitted but are included in the full paper version [1].

\section{MODEL DESCRIPTION AND PROBLEM FORMULATION}

We consider a finite user population $m$ users those transmit to a common base station over a shared channel. We identify each one by a unique i.d. number between 1 and $m$. We assume that time is slotted and all packets have the same length. Since we are interested in collision channels (such Aloha-like systems), a successful transmission occurs when only one user transmits in the current slot. We note that packets those are involved in a collision are backlogged and are scheduled for retransmission after a random time. Each user $i$ handles a buffer $Q_{i}$ (see Fig. 1) that carries packets arriving from high layers. Assume that packets arrive to the buffer $Q_{i}$ according to a Bernoulli process with fixed parameter $\lambda_{i}$. Hence $\lambda_{i}$ represents the normalized throughput demand (in number of packet per slot) for the user $i$ needed to hold the service reliability. Until we contraindicate, we assume that the buffers $Q_{i}$ have infinite capacity of storage, hence the loss probability due to buffer overflow is null. In the rest of the paper we refer to the vector of throughput demands by $\underline{\lambda}=\left(\lambda_{1}, \lambda_{2}, \ldots, \lambda_{m}\right)$.

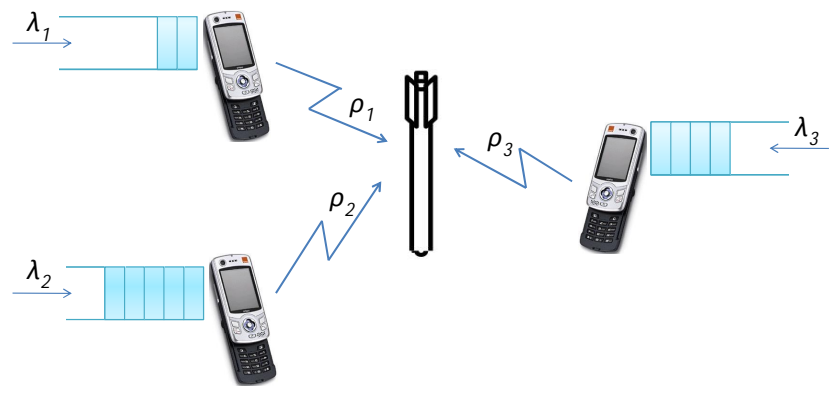

Figure 1: One central receiver and multiple transmitters. Each transmitter $i$ handles an infinitecapacity buffer and requires some minimum QoS $\lambda_{i}$.

REMARK 1. As mentioned already, this kind of systems are not analyzed in game theoretic framework before and we are trying to consider one important example of a non saturated system here. Indeed, a slotted aloha system with infi- 
nite buffer is non saturated as the users may not be busy in the beginning of each slot.

The underlying assumption of our user model is that users are selfish and do not cooperate in any manner in order to obtain their required throughput demands. We note clearly that the transmission rate of each users affects the throughput of other users. Each user $i$ fine-tunes its transmit probability so as to maximize its throughput (cannot exceed $\lambda_{i}$ ); A non cooperative game is then established. A user is said to be active if it has, in its buffer, at least one packet ready to be transmitted. Let $q_{i}$ denotes the probability that a user $i$ transmits on a given time slot and denote by $\underline{q}=\left(q_{1}, q_{2}, \ldots, q_{m}\right)$ the transmit probabilities vector. We characterize the state of the system by an $m$-dimensional vector. Let $\mathcal{M}=\{0,1\}^{m}$ represents the set of all $2^{m}$ subsets of $1,2 \ldots, m$. At each time slot, a subset $\mathcal{Z}$ of users is assumed to be active. The instantaneous number of active users is given by $|\mathcal{Z}(t)|=\sum_{i=1}^{m} \mathcal{Z}_{i}(t)$ which is the Hamming weight of $\mathcal{Z}$ [11]. The average throughput of user $i$ is then given by:

$$
\rho_{i}(\underline{q})=q_{i} \zeta\left(e_{i}\right) \sum_{\mathcal{Z} \in \mathcal{N} \backslash\{i\}} \zeta(\mathcal{Z}) \prod_{j \in \mathcal{Z}}\left(1-q_{j}\right),
$$

$e_{i}$ is the vector whose all entries equal zero but the ith which equals one and $\zeta\left(e_{i}\right)=\pi_{i}$ is the probability that user $i$ will be active. The equation (1) generalizes the throughput formula of collision channels where at most one successful transmission can occur per slot. Indeed, the activity probabilities of users present in the system are correlated, interdependent and still depend on time. Hence we shall write $\pi_{i}(\underline{q})$ instead of $\pi_{i}$.

The analysis of this system is quite complicated because of the complex nature of the formula (1). Even in symmetric user case this formula does not simplify to a good extent. In some papers, authors try to approximate this success probability under independence assumption. Under this assumption, the equation (1) would have simplified to [22]

$$
q_{i} \pi_{i}(\underline{q}) \Pi_{j \neq i}\left(1-q_{j} \pi_{j}(\underline{q})\right) .
$$

However unfortunately, this approximation is not good even for symmetric cases (please see figure 2). Hence the analysis of this system can not be simplified. This makes this study interesting and we carry out the analysis without independence assumption to obtain the required accurate analysis. We will start this job with first understanding the stability behavior of the system.

\section{STABILITY REGION AND RATE BAL- ANCE EQUATION}

Let Aloha $(\underline{\lambda}, q)$ represent the slotted aloha system with arrival rates $\underline{\lambda}$ and transmitting probabilities $q$. An Aloha $(\underline{\lambda}$, $\underline{q}$ ) system is an example of finite number of interacting queues. An Aloha system is stable whenever parameters related to it like, Buffer sizes etc., does not grow with probability one (please see [25] for exact definition). The stability analysis of such a system has been carried out in literature to a good extent.

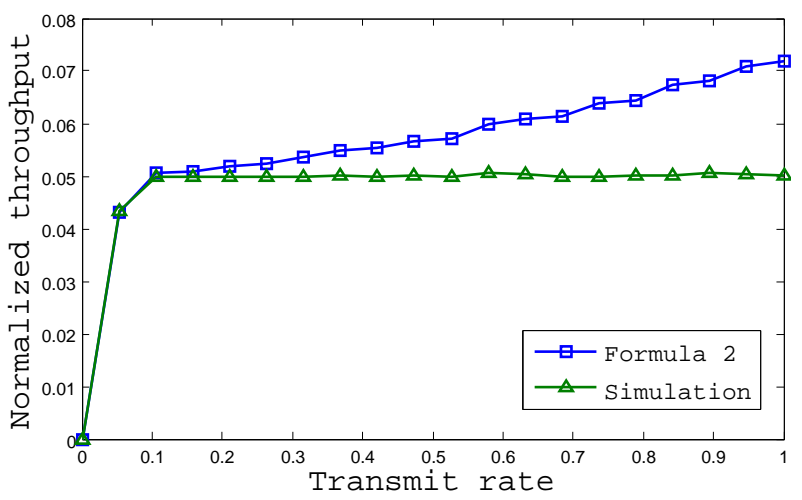

Figure 2: Figure illustrating the error while formula (2) based on the independence assumption is approximating the average throughput (success probability) of a symmetric Aloha system. Here, each user is assumed to have a fixed demand $\lambda=0.05$ (packets per slot). Transmit rate of 3 users is fixed to 0.5 whereas it is varying for the $4^{\text {th }}$ user.

Previously some studies have obtained explicit conditions for stability of Aloha $(\underline{\lambda}, \underline{q})$ for given values of $(\underline{\lambda}, \underline{q})$. Some of the interesting results in this direction are : the system is stable if it satisfies the condition (5) mentioned in the next section. In most of the other cases ([23], [25]), authors study the stability region, defined for a given transmit probability vector $q$, as the set of arrival rate vectors $\underline{\lambda}$ for which Aloha $(\underline{\lambda}, q)$ system is stable. While in some cases (for example $[24])$, they define the stability region as the set of those arrival rates $\lambda$ for which there exists a transmit vector $q$ with Aloha $(\underline{\lambda}, \underline{q})$ system being stable.

In this paper, we are interested in a different concept of stability region defined for a given arrival rates $\underline{\lambda}$ to be :

$$
\mathcal{Q}(\underline{\lambda}):=\left\{\underline{q} \in[0,1]^{m}: \text { Aloha }(\underline{\lambda}, \underline{q}) \text { is stable }\right\} .
$$

The reason for this kind of stability region becomes evident in the next section. In the following we obtain an alternative characterization of this stability region.

If an infinite buffer queue is stable it satisfies flow balance equations (as there can be no loss of packets): The input flow rate must be equal to the output flow rate. Here the arrival rate is the rate at which packets from higher layers arrives, i.e., $\lambda_{i}$ whereas the departure rate is exactly the success probability of user $i$ denoted by $P_{i}^{s u c c}$. Success probability $P_{i}^{\text {succ }}$ is same as $\rho_{i}(q)$ of equation (1) and these two terms are used to refer the same quantity. Hence our infinite buffer slotted aloha system is stable whenever

$$
\lambda_{i}=P_{i}^{\text {succ }}(\underline{q}) \text { for all } i .
$$

and hence we have:

Proposition 2. The stability region is then given by:

$$
\mathcal{Q}(\underline{\lambda})=\left\{\underline{q} \in[0,1]^{m}: \lambda_{i}=P_{i}^{\text {succ }}(\underline{q}) \text { for all } i\right\} .
$$

\section{NASH EQUILIBRIUM ANALYSIS}

As mentioned before, we study this problem using game theoretic frame work. It is interesting to note here that we will 
actually have a constrained game (will be more clear in the following paras). As a first step, we define the strategies of the underlying non cooperative game.

\subsection{Feasible strategy}

Each user $i$ has a demand in the form of throughput $\lambda_{i}$. Then, it fine-tunes its instantaneous transmit rate $q_{i}$ in order to fulfill this demand. Let $q_{-i}$ be the strategies vector of other users. To illustrate the meaning of feasible strategy we consider Fig. 3, which plots the payoff obtained by user $i$ as its transmit rate $q_{i}$ varies, when the other users fix their transmit strategies at $q_{-i}$. By payoff of user $i$ (with all the users attempting transmission at rates given by $q$ ), we mean the maximum demand of the user that can be met. Please note that this figure is not generated from any real example, but is only plotted to explain the concept of feasible strategy. From illustrative example in Fig. 3, one can see that user $i$ has, in general, several strategies that can guarantee the demand $\lambda_{i}$. Let $\Gamma_{i}\left(q_{-i}\right)$ be the set of all feasible strategies of user $i$, i.e., all strategies that can provide a throughput greater than or equal the demand $\lambda_{i}$. This requirement of the constraint on the strategies results then in a constrained game. We recall however that the obtained throughput of some given user $i$ cannot exceed its arrival rate $\lambda_{i}$.

Definition 3 (Definition 1). . A Nash equilibrium is a profile $q^{*}$ of feasible strategies such that no user can improve its ${ }^{*}$ utility by deviating unilaterally from its strategy to another chosen from the set of feasible actions $\Gamma_{i}\left(q_{-i}^{*}\right)$. Namely, $\left(q_{i}^{*}, q_{-i}^{*}\right)$ is a NE if and only if

$$
\rho_{i}\left(q_{i}^{*}, q_{-i}^{*}\right) \geq \rho_{i}\left(q_{i}, q_{-i}^{*}\right), \forall q_{i} \in \Gamma_{i}\left(q_{-i}^{*}\right) \text { and for all } i \text {. }
$$

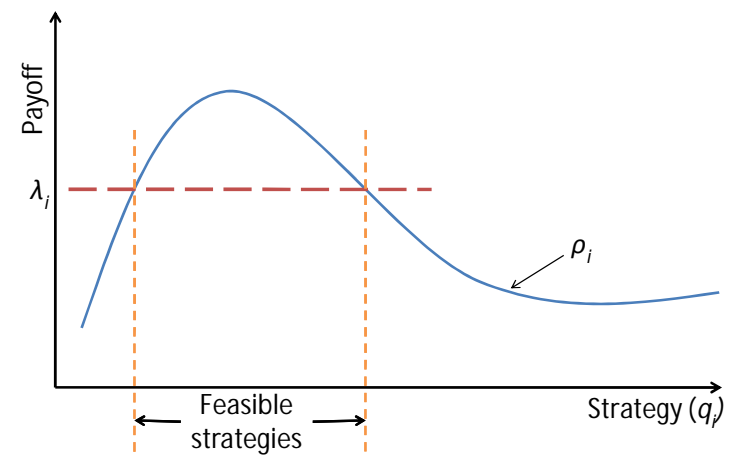

Figure 3: Feasible strategies of user $i$ under throughput demand $\lambda_{i}$ and average throughput $\rho_{i}$.

\subsection{Constrained Nash Equilibrium (CNE)}

We have a $m$-player constrained noncooperative game with throughputs as the utilities. Let $\mathcal{N}(\lambda)$ represent the region of all Constrained Nash equilibria.

The constrained game has a NE $q^{*}$ if and only if satisfies (3). Hence for the infinite buffer Slotted Aloha system, the region of $\mathrm{CNE}$ coincides with stability region, i.e.,

$$
\mathcal{Q}(\underline{\lambda})=\mathcal{N}(\underline{\lambda})
$$

\subsection{Existence of $\mathrm{CNE}$}

As mentioned in the previous section, for a given arrival rates $\underline{\lambda}$, the transmit rate vector $q$ is a CNE if and only if Aloha $(\underline{\lambda}, q)$ is stable. There are many results ([26], [23], [25] etc.) which establish the stability conditions, which thereby give the conditions for existence of NE. One such condition is (Theorem 1, [23]) : An Aloha $(\underline{\lambda}, q)$ system is stable if for all $i$,

$$
\lambda_{i}<q_{i} \Pi_{j \neq i}\left(1-q_{j}\right) .
$$

This establishes the existence of infinitely many NE:

$$
\mathcal{N}(\underline{\lambda}) \supset\left\{\underline{q} \in[0,1]^{m}: \lambda_{i}<q_{i} \Pi_{j \neq i}\left(1-q_{j}\right) \text { for all } i\right\},
$$

when the set on the right hand side is non empty.

For symmetric systems i.e., when all the users have equal demand, from [25, Corollary 4],

$$
\begin{aligned}
\mathcal{N}([\lambda, \cdots, \lambda]) \cap\left\{[q, \cdots, q] \in[0,1]^{m}\right\} & = \\
& \left\{[q, \cdots, q] \in[0,1]^{m}: \lambda<q(1-q)^{m-1}\right\} .
\end{aligned}
$$

\subsection{Energy Efficient Nash Equilibrium}

As seen in the previous section, there can in fact exist infinitely many of NE. From (6) the set of CNE, $\mathcal{N}$, contains an open set. In this section, we are interested in choosing a vector from $\mathcal{N}$ which will be (nearly) energy efficient for all the users.

Assume that $\mathcal{E}$ is the energy consumed for one single transmission. The expected energy consumption of user $i$ at any given slot is

$$
E_{i}=q_{i} \pi_{i} \mathcal{E}
$$

Empirically, the quantity $\pi_{i} q_{i}$, representing the apparent transmit rate, is increasing with $q_{i}$ (we notice this in almost all examples, see for example Fig. 4). Further, we note that when the demand is high the apparent transmit rate $q_{i} \pi_{i}$ tends towards the effective transmit rate $q_{i}$ since users become saturated. Hence the energy spent by user $q$ is approximately proportional to $q_{i}$, i.e.,

$$
E_{i} \propto q_{i}
$$

One can also support the above argument, by observing that, whenever the transmit probability of one of the users becomes less (by still remaining in stability region), the amount of collisions will be less and hence the overall energy spent by each and every user will be less.

Thus for obtaining an Energy Efficient NE (EEE), ideally we are interested in a point in $\mathcal{N}$, for which the transmit rate of all the users is the smallest possible. There can be many ways in obtaining such a Energy Efficient NE. In the following we define EEE as the the point where the product 
of all the transmit probabilities is minimized $^{2}$ :

$$
E E E:=\underset{\underline{q} \in \mathcal{N}}{\arg \min } \prod_{i=1}^{N} q_{i} .
$$

The set $\mathcal{N}$ is obviously bounded, by continuity arguments one can obtain an optimal point in the closure of the set $\mathcal{N}$. Even if the optimal point lies in the boundary of $\mathcal{N}$, one can chose a vector in $\mathcal{N}$ close to the optimal point so that its energy efficiency is arbitrarily close to that of the optimal point. Hence, there exists a $q^{*} \in \mathcal{N}$ which will be (nearly) energy efficient.

For symmetric users, let $q^{*}$ represent the smallest $q$ that satisfies $q(1-q)^{m-1}=\lambda$. Then note that $q^{*}:=\left[q^{*}, \cdots, q^{*}\right]^{T}$ achieves minimum in the definition of $\overline{E E E}$, but $q^{*} \notin \mathcal{N}$. Hence, any vector close to but not equal to $q^{*}$, will be (nearly) energy efficient.

\section{STOCHASTIC 'CNE' LEARNING ALGO- RITHMS}

In this section we will describe two stochastic iterative algorithms which converge to the efficient equilibrium point. Mobiles learn what strategy to adopt in order to obtain their required throughput. The first one is a semi distributed algorithm while the second one is a completely distributed and information less type.

\subsection{Best-response-based Distributed Algorithm (BRA)}

In [17] and [9], the authors proposed a best response-based algorithm that learn users the efficient equilibrium point for saturated case. One can extend the same iterative algorithm for non saturated (infinite buffer) case as follows.

Let $q_{i}^{t}, \pi_{i}^{t}$ respectively represent the transmit probability and activity probability of user $i$ at time slot $i$. Let

$$
\begin{aligned}
x_{i}^{t}(\underline{q}, \underline{\pi}) & :=\sum_{\mathcal{Z} \in \mathcal{N} \backslash\{i\}} \zeta(\mathcal{Z}) \prod_{j \in \mathcal{Z}}\left(1-q_{j}^{t}\right) \\
& \approx \prod_{j \neq i}\left(1-\pi_{j}^{t} q_{j}^{t}\right)
\end{aligned}
$$

be the idle probability of all users but the $i^{\text {th }}$ one at time slot $t$, which is approximated using independence assumption. Also, let $\epsilon_{i}^{t}$ be the update (learning) step at iteration $t$. At the beginning of each slot, we assume that the base station broadcasts the information $\left\{x_{i}^{t}, i \leq m\right\}$. The BRA version for the non saturated users is as below.

However the above extension of BRA algorithm has two important problems. It uses independence assumption in calculating idle probability (at BS), which as shown in Figure

\footnotetext{
${ }^{2}$ This can be understood well using the case of symmetric users. Say $q \in \mathcal{N}([\lambda, \cdots, \lambda])$. Let $j$ be user with smallest transmit attempt probability, i.e., such that $q_{j} \leq q_{i}$ for all $i$. Then $\left[q_{j}, \cdots, q_{j}\right] \in \mathcal{N},\left(q_{j}\right)^{m}<\Pi_{i \leq m} q_{i}$ and all the users will use lesser energy (when all users attempt transmission at rate $q_{j}$ on having a packet), than their corresponding energies associated with $\underline{q}$.
}

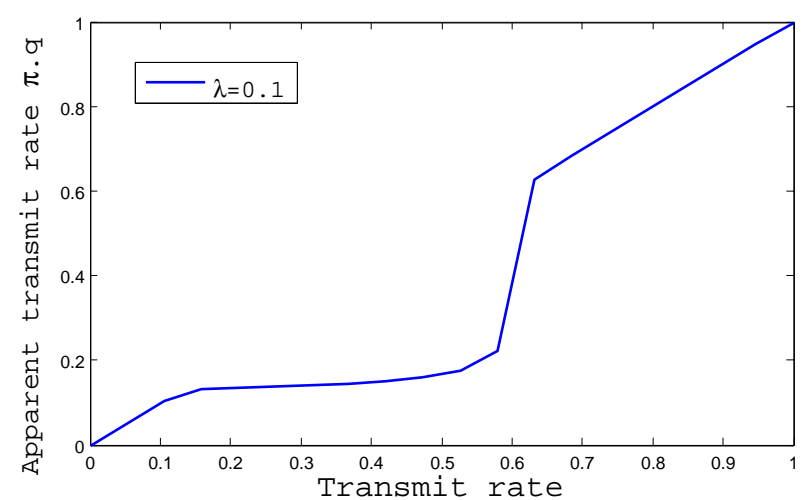

(a)

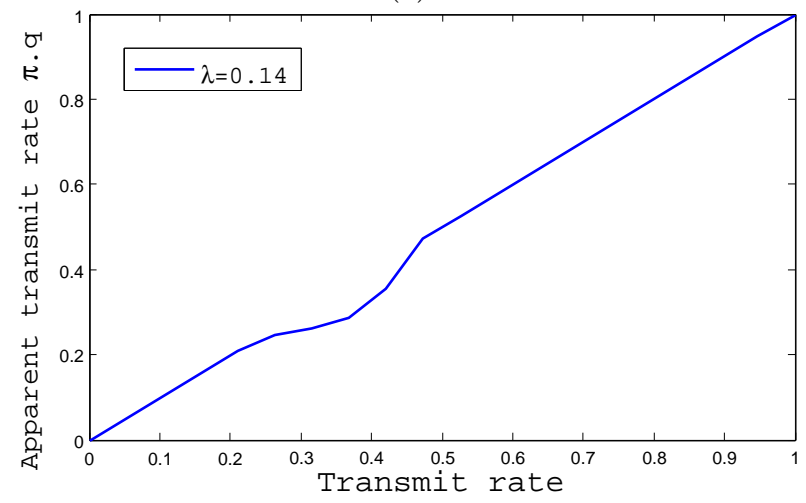

(b)

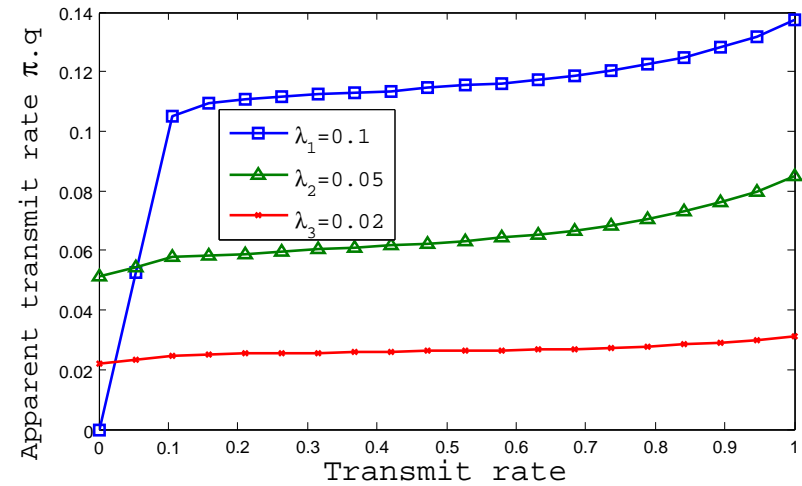

(c)

Figure 4: Fig. (a) and (b) show the apparent transmit rate as a function of the effective transmit rate for 3 symmetric users under different throughput demands. Fig. (c) shows the apparent transmit rate for 3 users with different throughput demands.

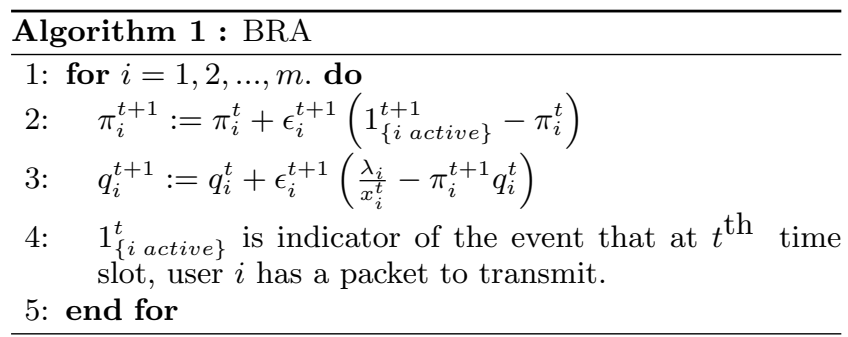


5 may not be a good approximation. If one were to estimate this quantity accurately, one needs to estimate $\zeta(\mathcal{Z})$, for each possible subset $\mathcal{Z}$, also iteratively as in the case of $\pi_{i}$. But this will complicate the algorithm considerably. Further, BRA is not a completely distributed algorithm and it requires the transmit rate information of all the users at every time step. This consumes valuable bandwidth and is also hard to implement. Further, this calls in for cooperation among users to share their private (transmit rate) information.

\subsection{Fully Distributed Throughput Predicting Algorithm (FDTPA)}

We now turn to develop a new algorithm that is completely distributed. We design an algorithm in which the users does not need any extra information except their own demand.

The key idea of the algorithm (2) is the following :

- The users can observe the sucess/failure of their own attempt to grab the collision channel.

- They can learn the effective throughput achieved by themselves by using the above observations.

- The users can estimate the deviation of their (current) throughput from their own demand $\left(\lambda_{i}\right)$ and adjusts their attempt/transmit rate to decrease this error to zero.

- On convergence, each user's effective throughput equals their respective demand. Thus the limit point achieves the demand (which is an important property of the $\mathrm{CNE})$.

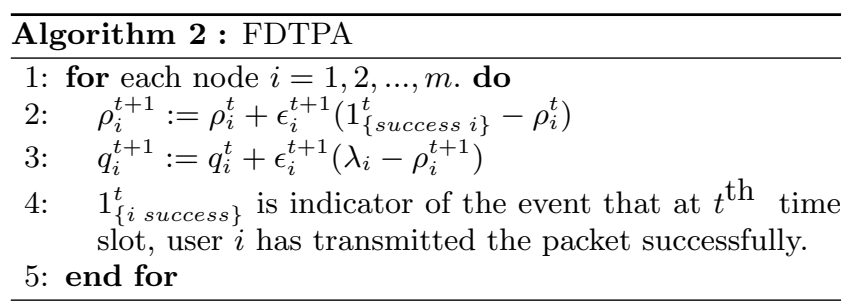

\subsubsection{Analysis}

We use ODE analysis to study the proposed FDTP algorithm. As a first step, we note that one can probably approximate the trajectory of the algorithm with the solution of following $\mathrm{ODE}^{3}$ system :

$$
\begin{aligned}
& \dot{\rho}_{i}(t)=P_{i}^{\text {succ }}(\underline{q}(t))-\rho_{i}(t) \\
& \dot{q}_{i}(t)=\lambda_{i}-\rho_{i}(t) \text { for all } i .
\end{aligned}
$$

We recall that $P_{i}^{\text {succ }}(\underline{q})$ represents the stationary probability of successful transmission at queue $i$ in a slotted Aloha $(\underline{\lambda}$, q).

${ }^{3}$ We are currently working towards obtaining the ODE approximation theorem.
Attractors . Since the ODE is approximating the trajectory of the FDTPA algorithm, its attractors give the limit points of the algorithm. From (9), any attractor $\left(\underline{q}^{*}, \underline{\rho}^{*}\right)$ of the ODE satisfies:

$$
P_{i}^{\text {succ }}\left(\underline{q}^{*}\right)=\rho_{i}^{*}=\lambda_{i}, \text { for all } i .
$$

Hence any attractor of the ODE (9) is a CNE and hence the limit point of FDTPA is a CNE.

\subsubsection{Initialization}

The ODE can have multiple attractors. It is already shown in the paper that there exists infinitely many CNEs (equation (6)). Hence the limit point of the algorithm depends mainly on the initial point. The algorithm converges to an attractor whenever it is initialized with a point close to it (a point in the region of attraction of the corresponding attractor). We are actually interested in converging to an EEE. The desirable EEE has lower value of $q_{i}^{*}$ for all users $i$ in comparison with the other attractors. Thus we will converge (with high probability) to the desired EEE if we initialize each $q_{i}^{0}$ with smallest possible value. Further from (1) at every $\mathrm{NE}, q_{i}^{*} \geq \lambda_{i}$. Hence the appropriate initialization values for the FDTPA algorithm are $q_{i}^{0}=\lambda_{i}$ and $\rho_{i}^{0}=\lambda_{i}$ for all $i$.

\subsubsection{Projected FDTPA}

Sometimes FDTPA can diverge because of the second iteration in the algorithm involving the updating of $q_{i}$ 's. The updated value of $q_{i}^{t+1}$ can go out of the window $[0,1]$ and this will cause the FDTPA to diverge. This can be taken care by the following projected FDTPA (with initializations $q_{i}=\rho_{i}=\lambda_{i}$ for all $i$ ):

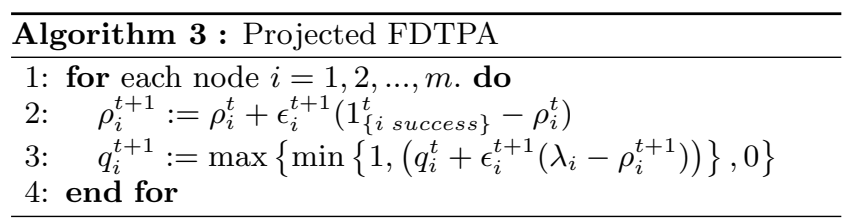

\section{NUMERICAL INVESTIGATION}

In the previous sections, we analyzed the Nash throughput for non saturated buffers and characterized the stability region. In the following, we present some analytical as well as some simulation results. We use among this section a discrete time simulator with Bernoulli process for packet generating.

\subsection{Stability and Nash Equilibrium Region}

We depict in Fig. 5 the individual throughput for 3 symmetric users when each one has a strict demand $\lambda=0.1$ (in packet per slot) and infinite buffer. The first main result is the validation, through simulation, of the existence of infinite number of Nash equilibria for this non saturated case. In this example, we restrict all three users to use same transmit probability $q$. In Fig. 5 we plot the simulated value of $P_{1}^{\text {succ }}$ and the approximating formula (2) versus the common transmit probability $q$. Indeed, we note (curve with triangle marks) that the throughput is increasing with the 
transmit rate. When the average throughput achieves the demand $\lambda$, we note that it becomes constant even if users continue to transmit more aggressively; This shows the existence of several Nash equilibria, potentially a continuum of NE. Over $q=0.6$, the throughput turns to decrease and vanishes when users become very aggressive (transmit at probability 1). This situation is similar to the prisoners dilemma, it shows in fact that Nash equilibrium is not efficient in some situations. Curve corresponding to the plot of average throughput using equation (2) seems to provide an accurate approximation only when users are transmitting at low or at high rate. This can be explained as it follows: On one hand, at low load the wireless network is not congested and then the collision probability is negligible. Therefore, the average throughput is only function of transmit rate and the approximation becomes accurate. On the other hand, when users become very aggressive, the collisions increase, the activity probability increases towards 1 , the system moves towards saturated case and henceforth the accuracy of the formula improves. In contrast to simulation, the approximation does not show existence of infinity NE points. To summarize, this kind of approximation is doubtful and has no interest since it seems to be so inaccurate that it can give wrong characterization of Nash equilibria points (equivalently stability region).

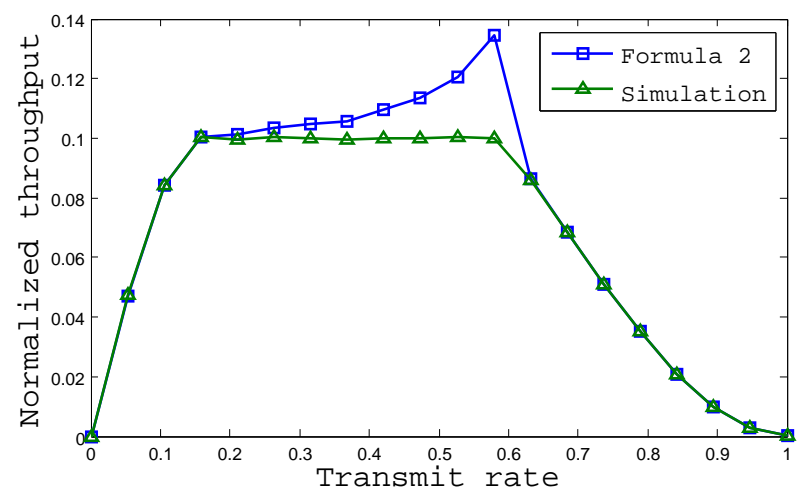

Figure 5: In this figure, we show the individual throughput for 3 symmetric users whose demand is fixed to $\lambda=0.1$, from both simulation and the approximation (using eq. 2).

Later we plot in Fig. 6 the variation of the activity probability (busy probability) of some tagged user for the example of Fig. 5. When user does not transmit at a rate that can guarantee its nominal demand, we note that $\pi=1$; This is due to the fact that the arrival rate is still greater than the departure rate. When the tagged user is transmitting at any rate in $[0.15,0.6]$, the activity probability is less than one; This interval corresponds well to the stability region. Through Fig. 5 and Fig. 6, we check easily that the region of Nash equilibria corresponds well to the stability region of the buffers. Further from the NE region characterization of Section 4, one can easily calculate that $\mathcal{N} \cap\left\{q \in[0,1]^{3}: q_{1}=q_{2}=q_{3}\right\}=[0.135,0.59]$, which is very close to the stability interval $[0.15,0.6]$ obtained from the simulations.

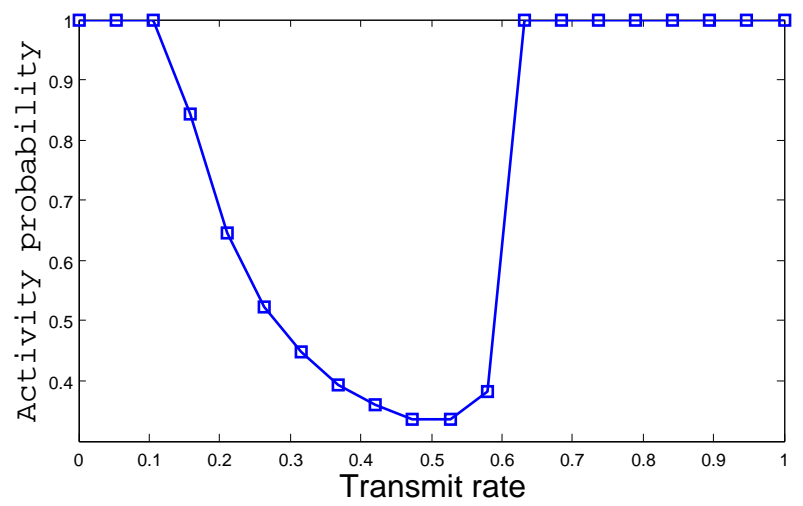

Figure 6: The activity probability versus transmit rate for 3 symmetric users whose demand is $\lambda=0.1$.

\subsection{Convergence of FDTPA and BRA algorithms}

We now turn to check and compare the efficiency of our two algorithms. We simulate in (Fig 7) the collision channel where users use respectively BRA (dashed line)and FDTPA (solid line). We consider a learning step $\epsilon=10^{-5}$. It is clear that information less stochastic scheme (FDTPA) tracks the desired Nash equilibrium as well as the version with partial information (BRA). We note similar trends when considering a variable learning step such as $\frac{1}{t+1}$ in Fig 8 .

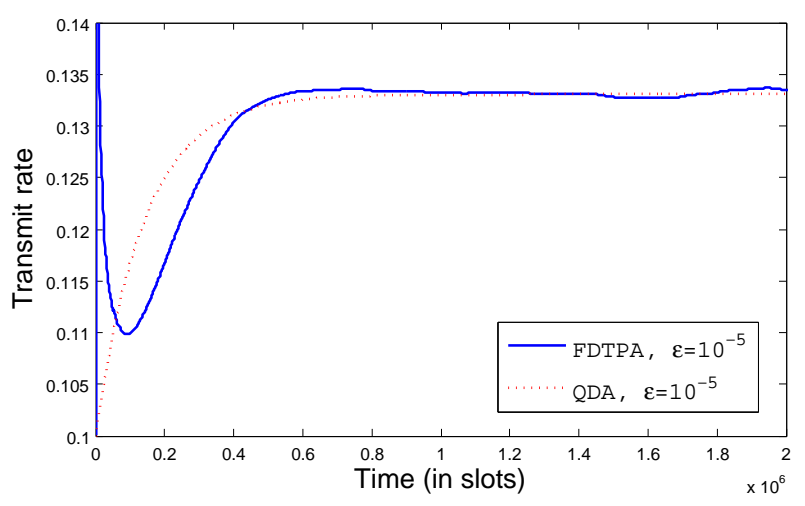

Figure 7: Simulation: In the case of small learning step $\left(\epsilon=10^{-5}\right)$, our stochastic algorithm performs as well as the BRA, either in term of accuracy and speed of convergence.

Fig 9-a and Fig 9-b show the crucial importance played by the initial conditions, in particular the initial transmit rate. Indeed if $q_{i}^{0}$ is not initialized judiciously the system can be absorbed by some non efficient equilibrium point. To avoid this problem, noting as said above that relation $\lambda_{i} \leq q_{i}$ always holds, initializing the transmit rate vector by the demand vector seems to provide a good start point. Another important factor that controls the speed of convergence, is the learning step, that should be chosen appropriately.

Next we simulate the behavior of the information less algorithm FDTPA in cases where no Nash equilibrium could exist. We depict in Fig. 10-a and Fig. 10-b the transmit rate and the average throughput respectively. The well known result of decentralized slotted aloha with selfish users ( [7], [6], $[2])$ is then obtained: Users transmit w.p. 1 which explain 
the throughput collapse and the congested situation of the whole system. A remark that might be interesting is that users are more aggressive as their demand is higher.

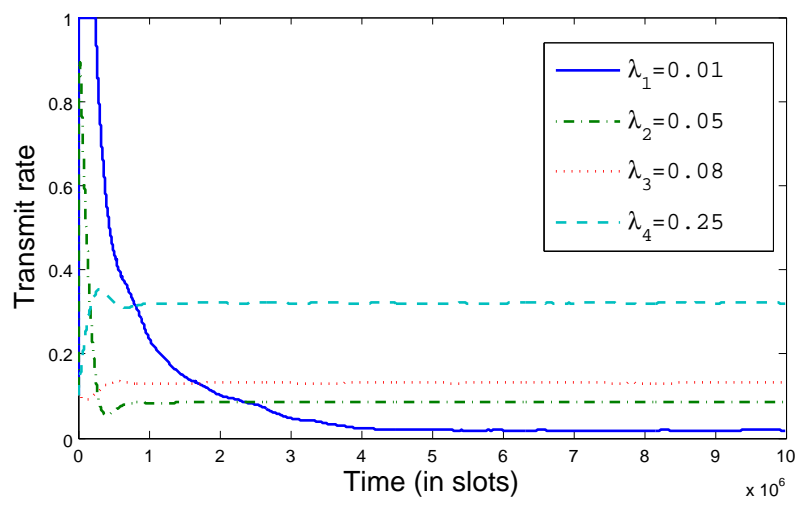

(a)

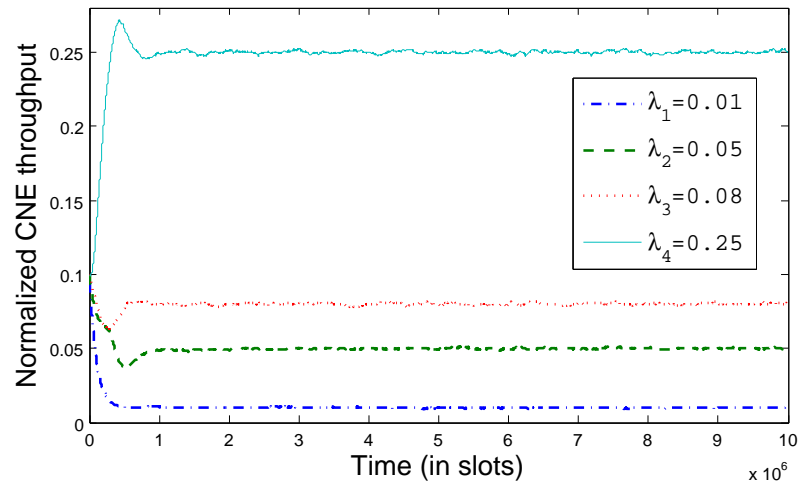

(b)

Figure 8: Here, the static learning step is taken to equal $\epsilon=10^{-5}, \lambda_{i}$ the throughput demand of user $i$. Fig $\mathbf{a}$ and $\mathbf{b}$ show the iterative evolution of the transmit rate and the average throughput until convergence to the EEE.

\subsection{Discussion}

The design of new protocols has face to several challenges and hard issues. For instance environment, bandwidth underutilization, energy constraint, evolution of hardware, resources allocation etc. Here we addressed an interesting problem that will drive progress in MAC layer protocols design. For saturated aloha system, authors in [9] presented a nice study for a saturated slotted aloha system. The main result is that when the throughput demands are within the demand feasible region, there exist exactly two Nash equilibria, with one strictly better (in terms of energy consumption) than the other for all users. However this seems not to be true for the same system with non saturated users. This can be simply explained as following: consider a NE $q^{1}$, a tagged user $i$ transmits at $q_{i}^{1}$ and is active w.p. $\pi\left(q_{i}^{1}\right)$, hence the transmit rate of user $i$ perceived by other users is $q_{i}^{1} \pi\left(q_{i}^{1}\right)$. Operating in the stability region, even if tagged user changes its transmit rate, its activity probability varies so as to keep the perceived transmit rate almost constant. Hence there exist many infinite NE. The real issue we should be careful with is the initialization point of transmit rates vector to converge to the $E E E$. Yet, the fluctuations might bring the system to some non efficient equilibrium. Setting the vector $q^{0}$ to $\underline{\lambda}$ is a judicious starting point that resolves the problem to a good extent.

Considering a fixed rate for every user can be of help to optimize the bandwidth utilization. On one hand, the minimum demand can be seen as the minimum QoS needed to keep the service reliability. On the other hand, this scheme can be seen as an alternative call admission control where the base station may be ensured that the system capacity is never exceeded.

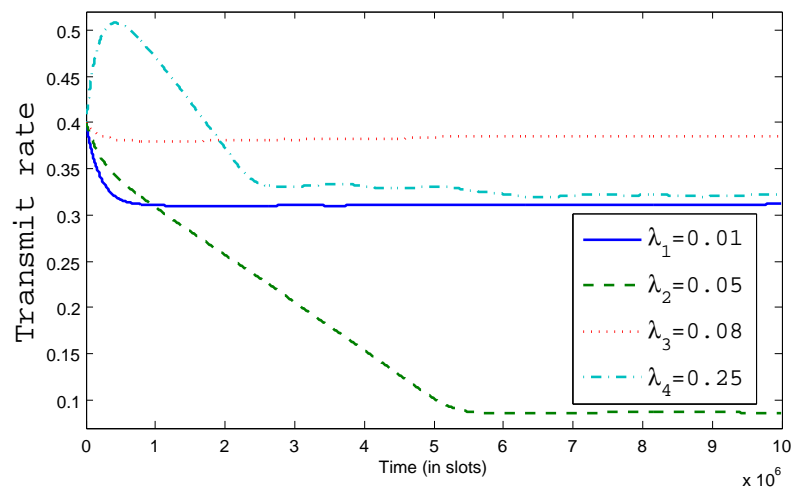

(a)

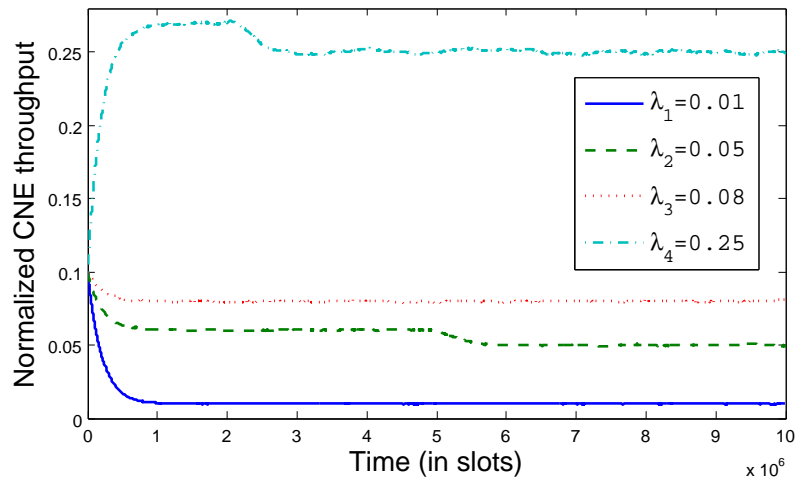

(b)

Figure 9: Importance of initializing the transmit rate is illustrated in Fig $a$ and $b$ where users converge to some non efficient CNE.

\section{CONCLUDING REMARKS}

We studied the throughput of collision channels, without saturation condition (usually assumed in the literature), where users have some strict QoS to fulfill. We noted that the achievable throughput is not affected by the users asymmetry and the region of equilibrium is larger than the saturated case. Indeed, we showed existence of infinite number of Nash equilibria. In addition to providing an energy efficiency analysis, characterizing the efficient CNE (EEE), we adapted the algorithm proposed in [17] and [9] taking into account the instantaneous saturation level of users in order to converge to EEE. However this algorithm suffers from many problems and implementation difficulties such as bandwidth consumption, doubtful estimation of previous 
transmit rate vectors and processing time. This motivated us to propose an information less stochastic distributed algorithm. We showed theoretically and through extensive simulations, the accuracy of the new algorithm as well as its speed of convergence.

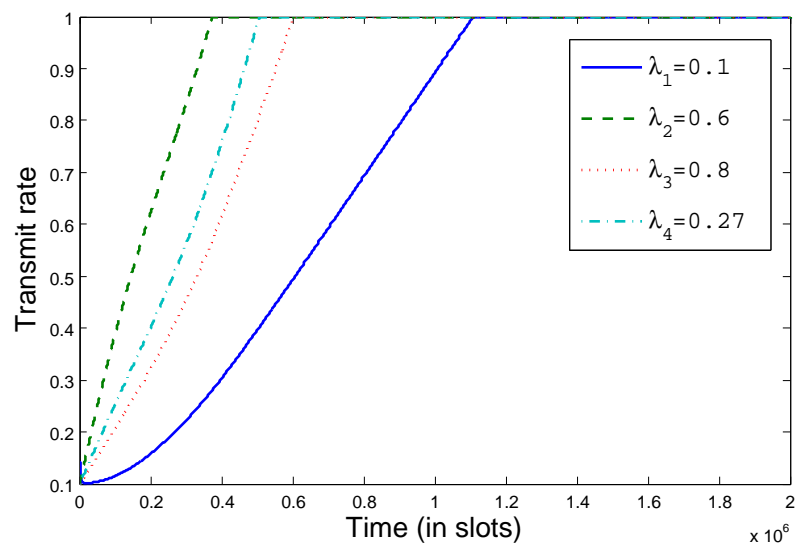

(a)

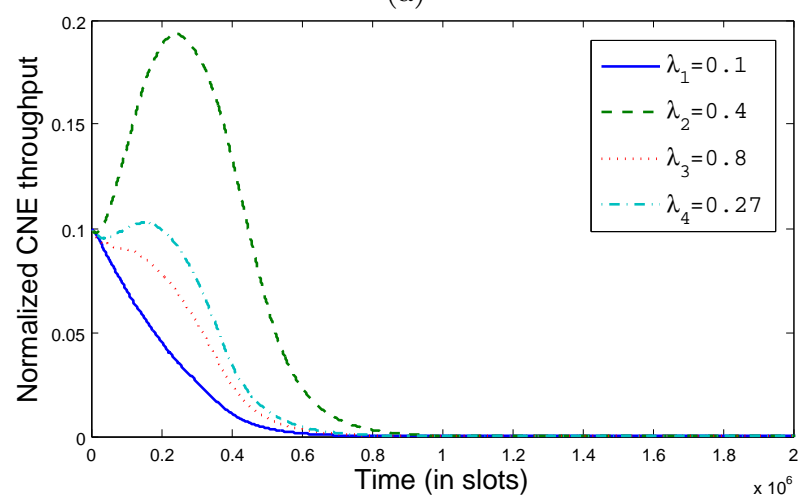

(b)

Figure 10: When a CNE does not exist users assist to a typical Prisoner's Dilemma phenomenon; Mobiles become very aggressive and transmit w.p. 1 (Fig a) which explain the throughput collapse (Fig b).

\section{REFERENCES}

[1] E. Sabir, R. El-Azouzi, V. Kavitha, Y. Hayel and E.H. Bouyakhf, "Stochastic Learning Solution for Constrained Nash Equilibrium Throughput in Non Saturated Wireless Collision Channels". http://lia.univ-avignon.fr/fileadmin/documents/ Users/Intranet/chercheurs/sabir/Collision-full.pdf

[2] R. El-Azouzi, E. Sabir, S. Benarfa, T. Jimenez and E. H. Bouyakhf, "Cooperative and noncooperative control for slotted aloha with random power level selection algorithms". Proc. valuetools/ACM,2007.

[3] G. Arslan, M. Fatih Demirkol and Y. Song, "Equilibrium efficiency improvement in MIMO interference systems: a decentralized stream control approach", IEEE Transaction on Wireless Communications, Volume 6, Issue 8, pages 2984-2993, August 2007.

[4] N. Abramson, "The Aloha system - another alternative for computer communications", AFIPS Conference Proceedings, Vol. 36, pages 295-298, 1970.

[5] L. G. Roberts, "Aloha packet system with and without slots and capture", Tech. Rep. Ass Note 8, Stanford Research Institute, Advance Research Projects Agency, Network Information Center, 1972.

[6] E. Altman, D. Barman, R El Azouzi and T. Jimenez, "A game theoretic approach for delay minimization in Slotted Aloha", ICC, 20-24, Paris, France, June 2004.

[7] E. Altman, R El Azouzi and T. Jimenez, "Slotted Aloha as a Stochastic Game with Partial Information", WiOpt'03, Sophia Antipolis, France, March 3-5, 2003.

[8] E. Altman, T. Boulogne, R. El Azouzi, T. Jiménez, L. Wynter, "A survey on networking games", 2006 Computers and Operations Research, Vol 33, Issue 2, pages 286-311, 2006

[9] I. Menashe and N. Shimkin, "Fixed-Rate Equilibrium in Wireless Collision Channels". Proc. Net-Coop'07, pp 23-32, Avignon, France.

[10] E. Altman, D. Barman, A. Benslimane and R. El-Azouzi, "Slotted Aloha with priorities and random power", in the proceedings of Networking, Ontario, Canada, pages 610-622, 2005.

[11] E. Altman, N. Bonneau and M. Debbah, "Correlated Equilibrium in Access Control for Wireless Communications", Networking 2006, Coimbra, Portugal.

[12] A. B. MacKenzie and S. B. Wicker, "Selfish users in Aloha: A game theoretic approach", IEEE VTC, fall, 2001.

[13] A. B. MacKenzie and S. B. Wicker, "Stability of Slotted Aloha with Multi-packet Reception and Selfish Users," Infocom, April 2003.

[14] Yiping Xing, Rajarathnam Chandramouli, "Stochastic learning solution for distributed discrete power control game in wireless data networks" IEEE/ACM Trans. Netw. 14(4): 932-944 (2008)

[15] D. Bertsekas and R. Gallager, "Data Networks", Prentice-Hall, 1992.

[16] J. J Metzner, "On improving utilization in ALOHA networks", IEEE Transaction on Communication COM-24 (4), 1976.

[17] Y. Jin and G. Kesidis, "Equilibria of a noncooperative game for heterogeneous users of an ALOHA network", IEEE Comm. Letters 6 (7), 282-284, 2002.

[18] J. H. Sarker, M. Hassan, S. Halme, "Power level selection schemes to improve throughput and stability of Slotted Aloha under heavy load", Computer Communication 25, 2002.

[19] P. S. Sastry, V. V. Phansalkar, and M. A. L. Thathachar, "Decentralized learning of Nash equilibria in multi-person stochastic games with incomplete information," IEEE Trans. Syst., Man, Cybernet., vol. 24, no. 5, pages 769Ü777, May 1994.

[20] Richard T.B.Ma, Vishal Misra and Dan Rubenstein, "Modeling and Analysis of Generalized Slotted-Aloha MAC Protocols in Cooperative, 
Competitive and Adversarial Environments", The 26th International Conference on Distributed Computing Systems (ICDCS 06), Lisbon, Portugal, 2006.

[21] R. O. LaMaire, A. Krishna and M. Zorzi, "On the randomization of transmitter power levels to increase throughput in multiple access radio systems", Wireless Networks 4, pp 263-277, 1998.

[22] T. Wan, A. U.H. Sheikh,"Analysis of finite population buffered slotted ALOHA protocolsusing tagged user analysis (TUA)", Globecom, Volume 3, Issue 1998, Pages:1652-1657, vol.3, 1998.

[23] R. Rao, A. Ephremides, "On the Stability of Interacting Queues in a Multiple-Access System", IEEE Transactions on Information Theory, Volume 34, Issue 5, Pages:918-930, September 1988.

[24] V. Anantharam, "The stability region of the finite-user slotted ALOHA protocol", IEEE Trans. Inform. Theory, vol. 37, pp. 535Ü540, May 1991.

[25] W. Szpankowski, "Stability conditions for some distributed systems", buffered random access systems, Adv. Appl. Probab, volume 26, pages:498-515, 1994.

[26] B. Tsybakov and W. Mikhailov, "Ergodicity of slotted ALOHA systems", Probl. Inform.

Transmission, volume 15, no. 4, pages:73-87, 1979. 